To appear in Connection Science

Vol. 00, No. 00, Month 20XX, 1-15

\title{
Swarmic Autopoiesis and Computational Creativity
}

\author{
Mohammad Majid al-Rifaie*, Frédéric Fol Leymarie, William Latham, Mark Bishop \\ [m.majid, ffl, w.latham, m.bishop] @gold.ac.uk \\ Department of Computing, Goldsmiths, University of London \\ London SE14 6NW, United Kingdom
}

(Received 00 Month 20XX; accepted 00 Month 20XX)

\begin{abstract}
In this paper two swarm intelligence algorithms are used, the first leading the 'attention' of the swarm and the latter responsible for the tracing mechanism. The attention mechanism is coordinated by agents of Stochastic Diffusion Search where they selectively attend to areas of a digital canvas (with line drawings) which contains (sharper) corners. Once the swarm's attention is drawn to the line of interest with a sharp corner, the corresponding line segment is fed into the tracing algorithm, Dispersive Flies Optimisation which 'consumes' the input in order to generate a 'swarmic sketch' of the input line. The sketching process is the result of the 'flies' leaving traces of their movements on the digital canvas which are then revisited repeatedly in an attempt to re-sketch the traces they left. This cyclic process is then introduced in the context of autopoiesis, where the philosophical aspects of the autopoietic artist are discussed.

The autopoetic artist is described in two modalities: gluttonous and contented. In the Gluttonous Autopoietic Artist mode, by iteratively focussing on areas-of-rich-complexity, as the decoding process of the input sketch unfolds, it leads to a less complex structure which ultimately results in an empty canvas; therein reifying the artwork's 'death'. In the Contented Autopoietic Artist mode, by refocussing the autopoietic artist's reflections on 'meaning' onto different constitutive elements, and modifying her reconstitution, different behaviours of autopoietic creativity can be induced and therefore, the autopoietic processes become less likely to fade away and more open-ended in their creative endeavour.
\end{abstract}

\section{Introduction}

Studies of the behaviour of social insects (e.g. ants and bees) and social animals (e.g. birds and fish) have proposed several new metaheuristics for use in collective intelligence. Natural examples of swarm intelligence that exhibit a form of social interaction are fish schooling, birds flocking, ant colonies in nesting and foraging, bacterial growth, animal herding, brood sorting (Bonabeau, Dorigo, and Theraulaz, 1999).

Although producing artistic works through the use of swarm intelligence techniques has been previously explored, this work explores the concepts of attention and autopoiesis through a type of collective intelligence, which emerges through the interaction of simple agents (representing the social insects and animals) in nature-inspired algorithms, namely Stochastic Diffusion Search (SDS) by Bishop (1989) and Dispersive Flies Optimisation (DFO) by al-Rifaie (2014).

In this work, SDS is deployed to enforce the idea of attention to area of the search space (digital canvas with line drawings) where there are more details (i.e. more lines or

\footnotetext{
* Corresponding author
} 
points); once the area of attention is identified, DFO through its flies, traces the points of the line selected and the flies' movements are visualised on the canvas. As attention moves from one area of the original line drawing to another, a sketch is produced which is the collective result of the SDS-led attention and millions of simple interactions facilitated by DFO algorithm.

In the last couple of years, several research work have been conducted utilising the two aforementioned swarm intelligence algorithms (e.g. al-Rifaie and al-Rifaie (2016); al-Rifaie and Aber (2016); al-Rifaie, Aber, and Hemanth (2015); Bedi, Bhasin, Mittal, and Chatterjee (2014); Salman, Ahmad, and Omran (2015)). Additionally, the authors have been exploring the artistic capabilities of swarm intelligence techniques in several publications (al-Rifaie and Bishop (2013a b) ), along with some philosophical arguments on the computational creativity of such systems (e.g. al-Rifaie, Cropley, Cropley, and Bishop (2016); Bishop and Al-Rifaie (2016)).

In the following sections, initially a brief overview of some of the work in generative art and swarm intelligence is provided. Subsequently, the two swarm intelligence algorithms deployed here are presented. Afterwards, attention and tracing mechanisms associated with the two swarm intelligence algorithms are presented, thus providing details on the performance of the computer-generated nature-inspired attentive swarms in re-interpreting the original line drawings. Finally, the concept of autopoietic swarmic artist is discussed in the philosophical context of autopoiesis.

\section{Generative art and swarm intelligence}

Among the many works in the field of generative art are research on swarm painting (Aupetit, Bordeau, Monmarche, Slimane, and Venturini, 2004, Moura and Ramos, 2007, Urbano, 2005, 2006), which includes, ant colony paintings (Greenfield, 2005; Monmarche, Aupetit, Bordeau, Slimane, and Venturini, 2003; Semet, O'Reilly, and Durand, 2004) and other multi-agent systems, including RenderBots (Schlechtweg, Germer, and Strothotte, 2005) as well as the particle-based non-evolutionary approach of Loose and Sketchy Animation (Curtis, 1998).

In most of such swarm-based work (Aupetit et al., 2004; Greenfield, 2005; Moura and Ramos, 2007; Urbano, 2005, 2006), the painting process does not re-work an initial drawing, but rather focuses on presenting "random artistic patterns", somewhere between order and chaos (Urbano, 2006). Other classes of research by (Curtis, 1998; Schlechtweg et al. 2005) are based on reworking an initial drawing. There is a significant number of related papers in the area of non-photorealistic rendering; particularly, many papers approach drawing and painting using an optimisation framework. Furthermore, particles have been used for stippling and to achieve other aesthetic styles in numerous papers. The work by Turk and Banks (1996) is an early example of optimising particle positions to control a stroke-based rendering. Hertzmann (Hertzmann, 2001) optimised a global function over all strokes using a relaxation approach. Collomosse and Hall (2005) have used a global genetic algorithm to define a rendering algorithm. More recently, Zhao and Zhu (2011) deployed an optimisation-based approach to study the stroke placement problem in painterly rendering, and presented a solution named stroke processes, which enables intuitive and interactive customisation of painting styles. 


\section{Communication in social systems}

Communication, as a social interaction or information exchange observed in social insects and social animals plays a significant role in all swarm intelligence algorithms, including SDS and DFO. Although in nature it is not only the syntactical information that is exchanged between the individuals but also semantic rules and beliefs about how to process this information (Kennedy, Eberhart, and Shi, 2001), in typical swarm intelligence algorithms only the syntactical exchange of information is taken into account.

In the study of the interaction of social insects, two important elements are the individuals and the environment, which result in two integration schemes: the first is the way in which individuals interact with each other, and the second is the interaction of the individuals with the environment (e.g. through pheromones) (Bonabeau, Dorigo, and Theraulaz, 2000). Self-interaction between individuals is carried out through recruitment strategies and it has been demonstrated that, typically, various recruitment strategies are used by ants (Holldobler and Wilson, 1990) and honey bees. These recruitment strategies are used to attract other members of the society to gather around one or more desired areas, either for foraging purposes or for moving to a new nest site.

In general, there are many different forms of recruitment strategies used by social insects; these may take the form of global or local strategies; one-to-one or one-to-many communication; and the deployment of stochastic or deterministic mechanisms. The nature of information sharing varies in different environments and with different types of social insects. Sometimes the information exchange is quite complex where, for example it might carry data about the direction, suitability of the target and the distance; or sometimes the information sharing is simply a stimulation forcing a certain triggered action. What all these recruitment and information exchange strategies have in common is distributing useful information throughout their community (de Meyer, Nasuto, and Bishop, 2006).

However, in many hive-based or flock-based agent systems - similar to the ones deployed in this work - the benefits of memory and communication seem obvious, but as argued in Schermerhorn and Scheutz (2009), these abilities are not beneficial in every environment, depending on the way resources are clustered throughout that environment and whether the quality of the food sources is sufficiently high.

The algorithms reported in this paper both rely on memory and communication to enable the agents to explore various parts of the search space; albeit the communication methods outlined herein are less greedy than the one presented in Schermerhorn and Scheutz (2009).

\subsection{From social interactions to intelligent reasoning}

The parable of 'The Blind Men and the Elephant' suggests how social interactions can lead to a more intelligent reasoning. This famous tale, set in verse by John Godfrey Saxe (Saxe, Lathen, and Chief, 1882) in the 19th century, characterises six blind men approaching an elephant. They end up having six different ideas about the elephant, as each person has experienced only one aspect of the elephant's body: wall (elephant's side), spear (tusk), snake (trunk), tree (knee), fan (ear) and rope (tail). The moral of the story is to show how people build their beliefs by drawing them from incomplete information, derived from partial knowledge about the world (Kennedy et al., 2001). If the blind men had been communicating about what they were experiencing, they would have possibly come up with the conclusion that they were exploring the heterogeneous 
qualities that make up an elephant.

\section{Swarm intelligence algorithms}

This section describes the two swarm intelligence algorithms used in this work (i.e. Stochastic Diffusion Search or SDS and Dispersive Flies Optimisation or DFO) and details the process through which they operate.

\subsection{Stochastic Diffusion Search (SDS)}

This section introduces SDS whose performance is based on simple interactions of agents (Bishop, 1989). This algorithm is inspired by one species of ants, Leptothorax acervorum, where a 'tandem calling' mechanism (one-to-one communication) is used. The forager ant that finds the food location recruits a single ant upon its return to the nest, as such the location of the food is physically publicised (Moglich, Maschwitz, and Holldobler, 1974).

The SDS algorithm commences a search or optimisation by initialising its population and then iterating through two phases (Algorithm 1).
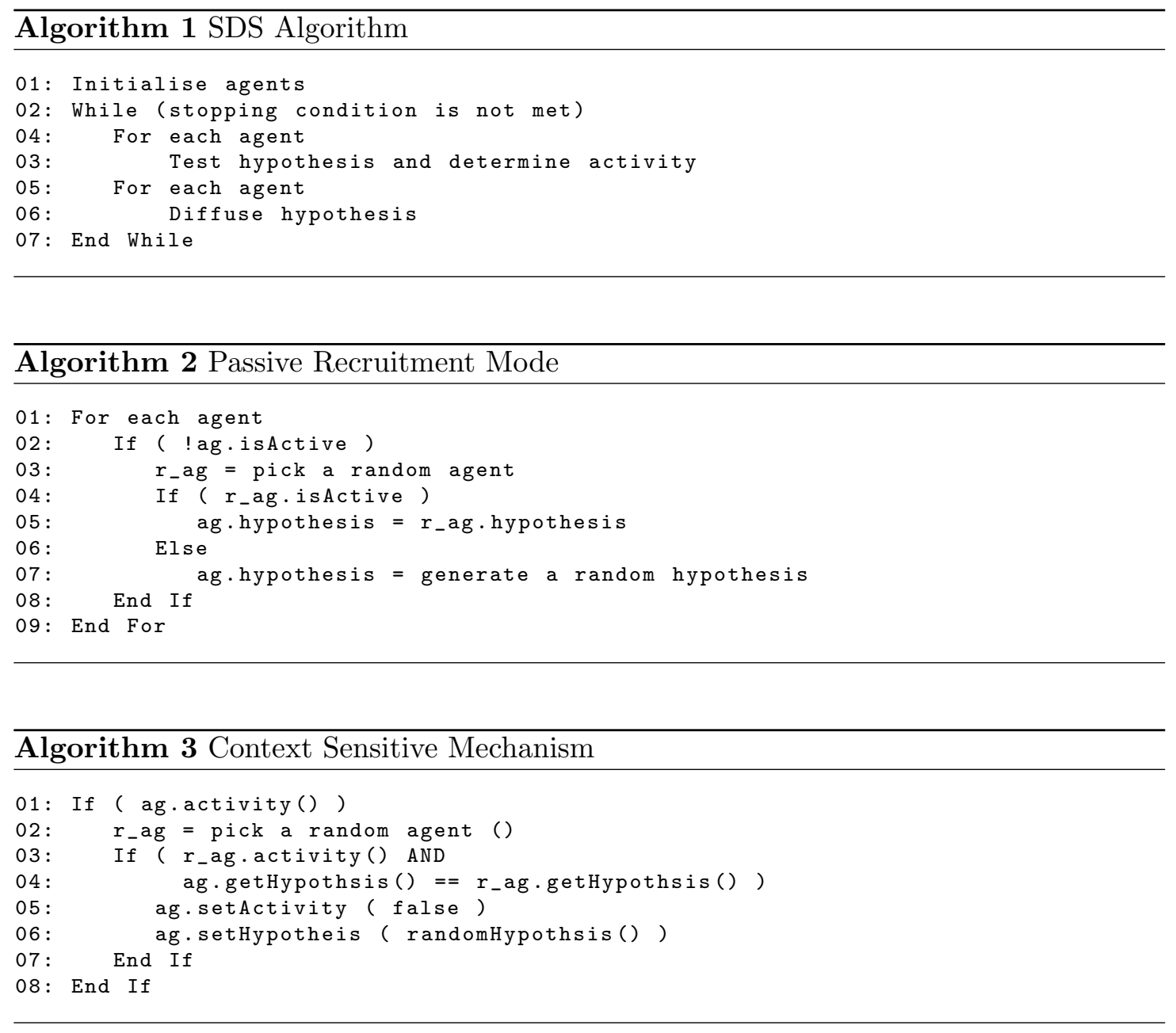
In the test phase, SDS checks whether the agent hypothesis is successful or not by performing a hypothesis evaluation which returns a Boolean value. Later in the iteration, contingent on the precise recruitment strategy employed (in the diffusion phase), successful hypotheses diffuse across the population and in this way information on potentially good solutions spreads throughout the entire population of agents. In other words, each agent recruits another agent for interaction and potential communication of hypothesis.

In standard SDS, a passive recruitment mode is employed (Algorithm 2). In this mode, if the agent is inactive, a second agent is randomly selected for diffusion; if the second agent is active, its hypothesis is communicated (diffused) to the inactive one. Otherwise there is no flow of information between agents; instead a completely new hypothesis is generated for the first inactive agent at random. Therefore, recruitment is not the responsibility of the active agents.

Additionally, in this work, context sensitive mechanism (Algorithm 3) is used, such that if the selected agent is both active and has the same hypothesis, the selecting agent is set inactive and is made to pick a random hypothesis. This mechanism frees up some of the resources in order to have a wider exploration throughout the search space as well preventing cluster size from overgrowing, while ensuring the formation of large clusters in case there exists a perfect match or good sub-optimal solutions.

\subsection{Dispersive Flies Optimisation (DFO)}

DFO is an algorithm inspired by the swarming behaviour of flies hovering over food sources. As detailed by al-Rifaie (2014), the swarming behaviour of flies is determined by several factors including the presence of threat which disturbs their convergence on the marker (or the optimum value). Therefore, having considered the formation of the swarms over the marker, the breaking or weakening of the swarms is also noted in the proposed algorithm.

In other words, the swarming behaviour of the flies in DFO consists of two tightly connected mechanisms, one is the formation of the swarms and the other is its breaking or weakening. The algorithm and the mathematical formulation of the update equations are introduced below.

The position vectors of the population are defined as:

$$
\vec{x}_{i}^{t}=\left[x_{i 1}^{t}, x_{i 2}^{t}, \ldots, x_{i D}^{t}\right], \quad i=1,2, \ldots, N P
$$

where $t$ is the current time step, $D$ is the dimension of the problem space and $N P$ is the number of flies (population size).

In the first generation, when $t=0$, the $i^{t h}$ vector's $j^{t h}$ component is initialised as:

$$
x_{i d}^{0}=x_{\min , d}+r\left(x_{\max , d}-x_{\min , d}\right)
$$

where $r$ is a random number drawn from a uniform distribution on the unit interval $U(0,1) ; x_{\min }$ and $x_{\max }$ are the lower and upper initialisation bounds of the $d^{\text {th }}$ dimension, respectively. Therefore, a population of flies are randomly initialised with a position for each flies in the search space.

On each iteration, the components of the position vectors are independently updated, taking into account the component's value, the corresponding value of the best neighbouring fly (consider ring topology) with the best fitness, and the value of the best fly 
in the whole swarm:

$$
x_{i d}^{t}=x_{n b_{i}, d}^{t-1}+\mathrm{U}(0,1) \times\left(x_{s b, d}^{t-1}-x_{i d}^{t-1}\right)
$$

where $x_{n b_{i}, d}^{t-1}$ is the value of the neighbour's best fly of $\overrightarrow{x_{i}}$ in the $d^{t h}$ dimension at time step $t-1 ; x_{s b, d}^{t-1}$ is the value of the $s$ warm's best fly in the $d^{t h}$ dimension at time step $t-1$; and $\mathrm{U}(0,1)$ is the uniform distribution between 0 and 1 .

The algorithm is characterised by two main components: a dynamic rule for updating flies position (assisted by a social neighbouring network that informs this update), and communication of the results of the best found fly to other flies.

As stated earlier, the swarm is disturbed for various reasons; one of the positive impacts of such disturbances is the displacement of the disturbed flies which may lead to discovering a better position. To consider this eventuality, an element of stochasticity is introduced to the update process. Based on this, individual components of flies' position vectors are reset if the random number, $r$, generated from a uniform distribution on the unit interval $U(0,1)$ is less than the disturbance threshold or $d t$. This guarantees a proportionate disturbance to the otherwise permanent stagnation over a likely local minima. Algorithm 4 summarises the DFO algorithm 1 .

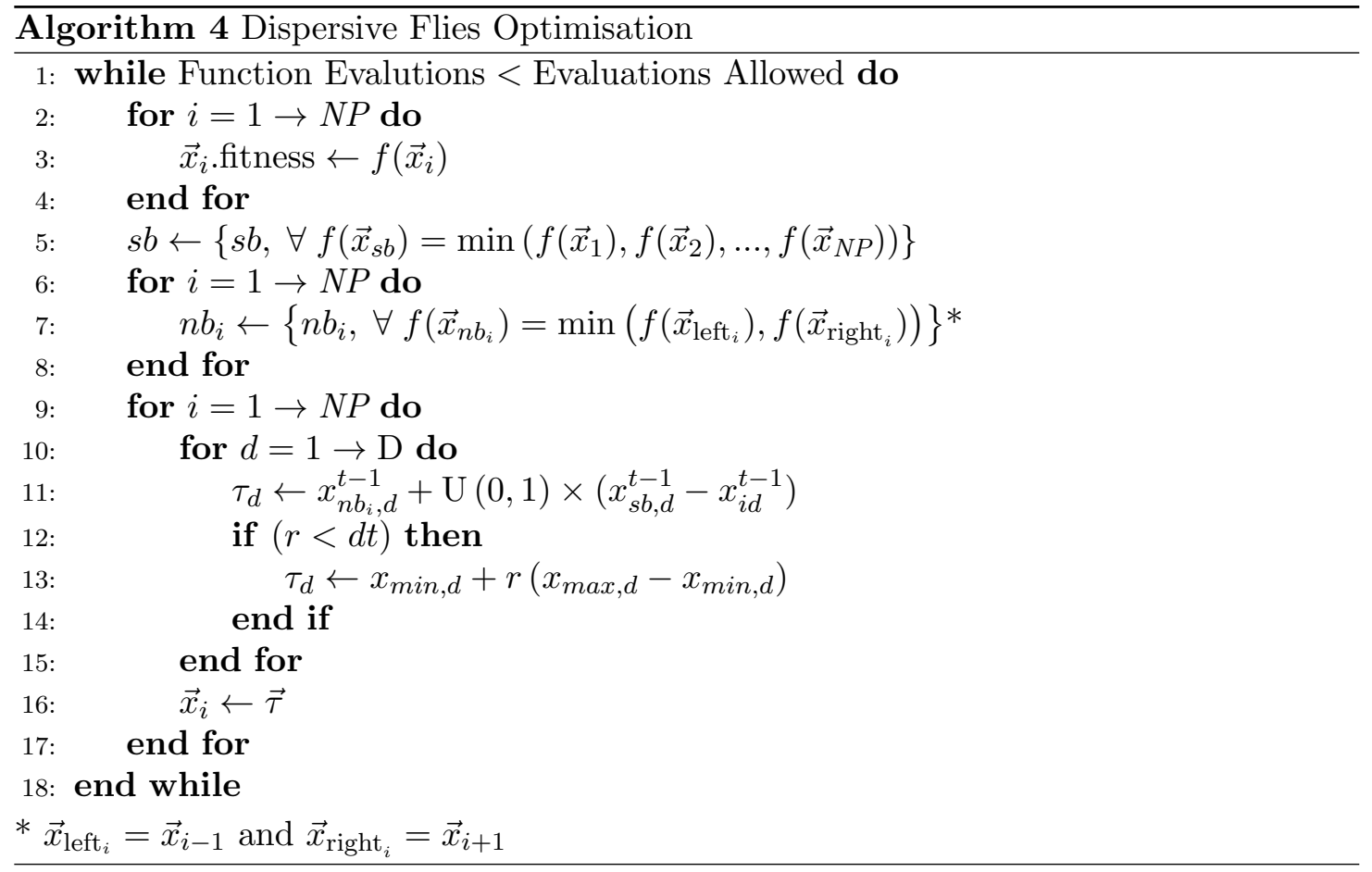

In summary, DFO is a simple numerical optimiser over continuous search spaces. DFO is a population based stochastic algorithm, originally proposed to search for an optimum value in the feasible solution space. Despite the algorithm's simplicity, it is shown that DFO outperforms the standard versions of the well-known Particle Swarm Optimisation, Genetic Algorithm (GA) as well as Differential Evolution (DE) algorithms on an extended set of benchmarks over three performance measures of error, efficiency and reliability (al-Rifaie, 2014). It is shown that DFO is more efficient in $84.62 \%$ and more reliable in

${ }^{1}$ The source code can be downloaded from the following page: http://doc.gold.ac.uk/ map01mm/DFO/ 
$90 \%$ of the 28 standard optimisation benchmarks used; furthermore, when there exists a statistically significant difference, DFO converges to better solutions in $71.05 \%$ of problem set. In addition to theoretical research on this algorithm, DFO has recently been applied to medical imaging (al-Rifaie and Aber, 2016).

In the work presented here, DFO is used for its tracing mechanism and its $d t$ parameter is set to 0 .

\section{Attention \& creativity in the swarms}

In this section, a simple attention mechanism, which is controlled by the SDS algorithm is detailed. This is followed by the process through which the DFO algorithm utilises the output of the SDS-led attention to visualise the flies movements on the digital canvas which in turn produces the final sketch rendered by the swarms.

\subsection{Attention mechanism}

The input digital image consists of line drawings (Fig. 1) where each line is formed up of a series of points. The swarms' attention in this work is directed towards the sharpness of the corners or curves found in the line drawings. In other words, the corner's (or a curve's) sharpness forms the agents' fitness values, $f_{i,(x, y)}$, where $i$ is the agent number and $(x, y)$ is the coordinate of the point taken from a certain line in the search space (input image). The agents' hypotheses in this scenario are the $(x, y)$ coordinates which are initially selected randomly from the points on the digital canvas. Fitness of an agent is calculated by taking two points from either side of the hypothesis (i.e. $(x, y)$ coordinate) with distance $d$ (which influence how wide or narrow an angle the system is looking for). Assuming the point referring to the coordinate of the hypothesis is $p_{h}$ and the two points on either left and right sides, $p_{l}$ and $p_{r}$, the fitness is the angle formed on $p_{h}$ when lines are drawn from $p_{h}$ to $p_{l}$, and from $p_{h}$ to $p_{r}$. The images in Fig. 2 show the initialisation of agents on various points on the line drawing, and the result of running one SDS test phase which will be explained later.

As mentioned earlier in Section 4.1, each agent has two components: status, which is a boolean value and hypothesis. The hypothesis of each agent in this work is the $(x, y)$ coordinate which is used to calculate the fitness, $f_{i,(x, y)}$, of the agents located at any particular point on the line drawing.

After randomly initialising the agents throughout the search space, in order to determine the status of an agent, $i$, within the swarm (test phase), its fitness, $f_{i}$, is calculated as explained above and another agent, $r$, is randomly selected; if $f_{i}$ is better than $f_{r}$ (i.e. the agent $i$ is located on a sharper corner/curve), agent $i$ is set active, otherwise inactive.

In the diffusion phase, each inactive agent randomly picks another one. If the randomly selected agent is active, the inactive agent adopts the hypothesis of the active one. However, if the selected agent is inactive, the selecting agent generates a random hypothesis $(x, y)$ from the search space. Furthermore, as in context-sensitive mechanism if the agent is active, it selects another agent randomly, if the selected agent is active and has the same hypothesis, the selecting agent becomes inactive and selects another hypothesis randomly from the search space. This mechanism allows the agents to maintain their exploration "attitude".

After $n$ number of test-diffusion phases cycles, the biggest cluster of the agents is identified and the associated line (where point $p_{h}$ is located on) is singled out. Once the $(x, y)$ coordinate of the point is retrieved, the starting and end points of the line is 
Figure 1. Input images: series of points forming line drawings. The image on the left is after one of Matisse's sketches, and the image on the right is after one of de Kooning's.
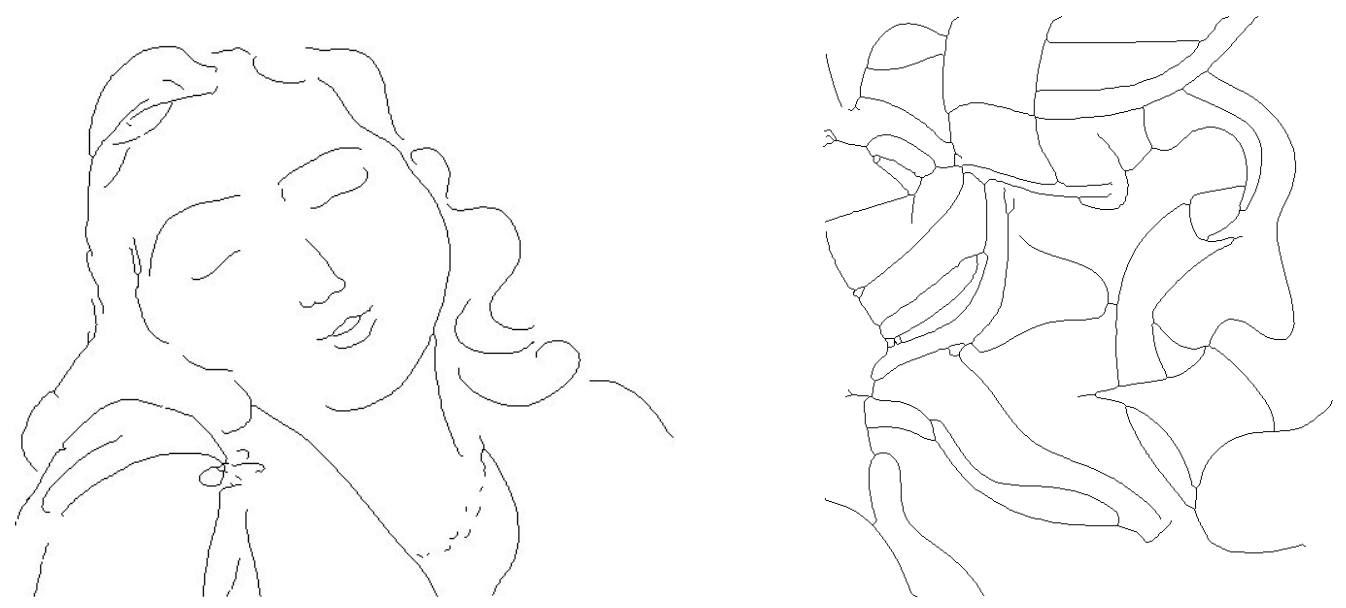

Figure 2. (a) Agent's initialisation: Each agent is initialised at a random point on the line drawing. The agent colour is red which indicate that agents are initially inactive. (b) Agents becoming active are highlighted in green.

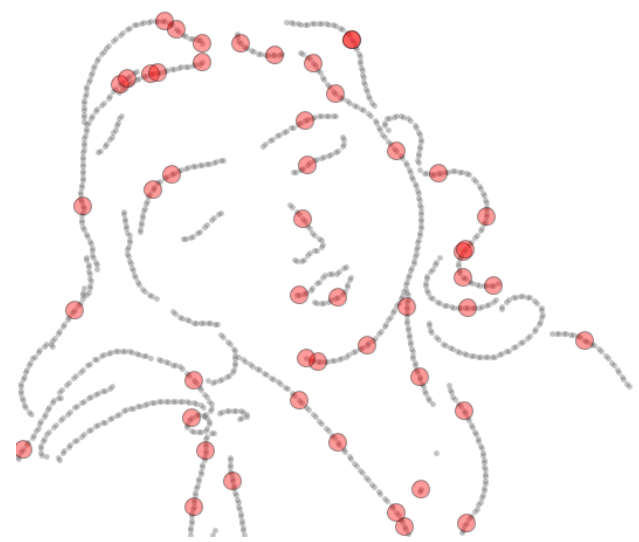

(a)

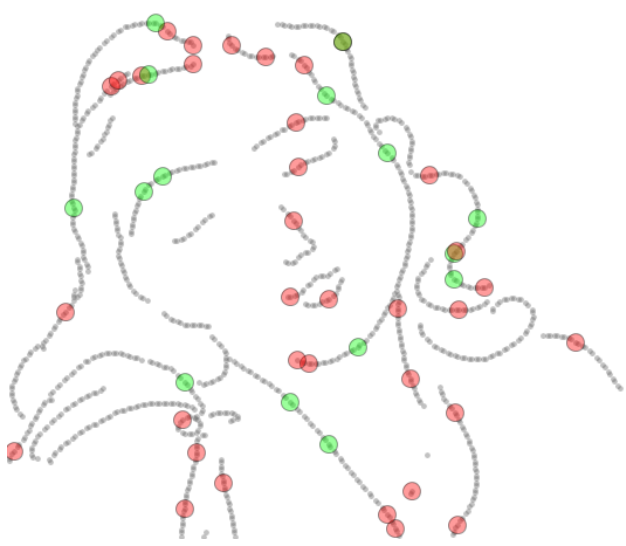

(b)

extracted and a string of $(x, y)$ coordinates from starting to end point of the line is passed on to the DFO flies to trace the points of the line, one by one. Fig. 3 shows the convergence of agents after $n$ iterations of test and diffusion phases, therefore picking a line to be fed into the other swarm intelligence algorithm, DFO, for the tracing mechanism.

\subsection{Tracing mechanism}

The points constituting the lines of the line drawing are treated as targets by the flies of DFO algorithm. Thus, the flies aim to trace these points one at a time until reaching the end of the line (the algorithm tries to minimise the distance between the flies' positions and the points it aim to track). Flies's movement is visualised on the canvas (i.e. trajectory of the flies moving from position $\left(x_{0}, y_{0}\right)$ to $\left(x_{1}, y_{1}\right)$ and so forth).

Input to DFO algorithm is a series of points forming up a line (whose starting and end points are extracted as mentioned above). The algorithm is then instructed to trace 
Figure 3. Agents convergence

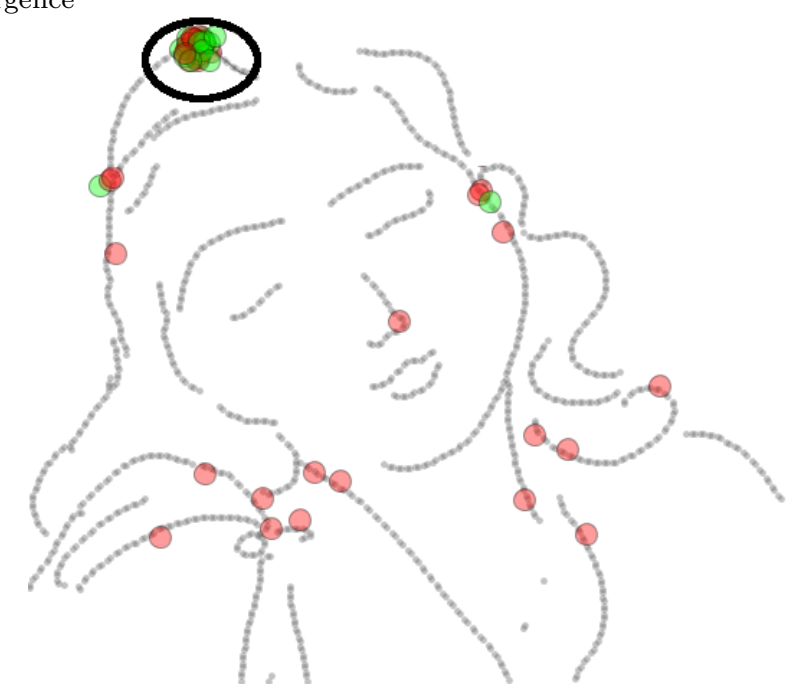

Figure 4. Visual Display of the System. Left: visualising DFO's tracing mechanism; middle, the new generation of points produced by the flies; right: attention of the SDS agents
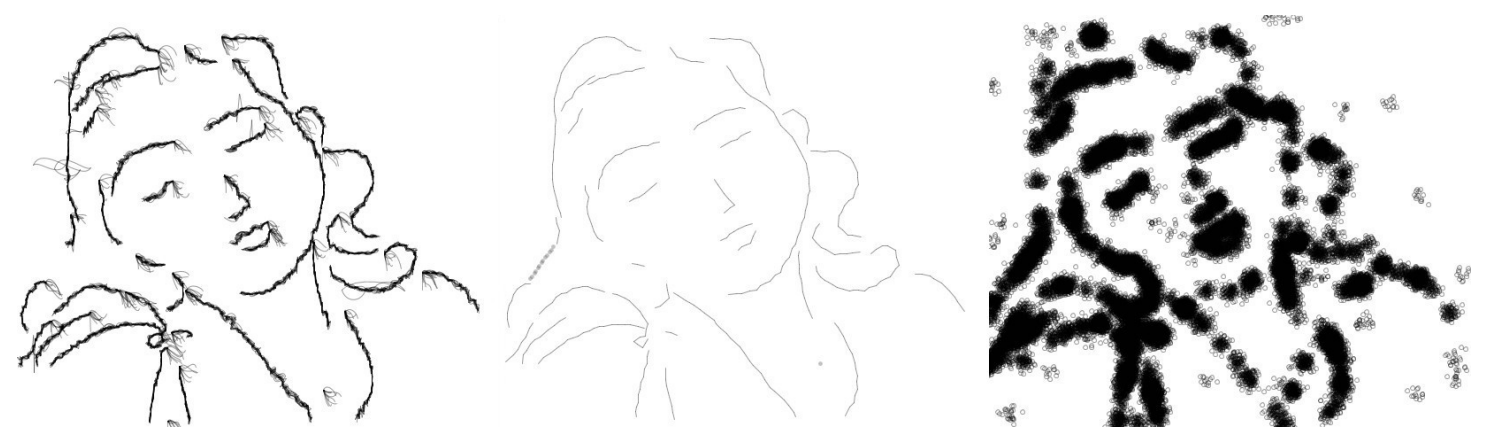

the line from the beginning to the end. Once the line is traced, it is removed from the search space and the other lines are considered one by one according to the attention mechanism deployed.

As shown in Fig. 4, the canvas is divided in three sections: on the left, the result of DFO's tracing mechanism can be viewed (i.e. the process through which the DFO flies trace the points of the lines and their movements are illustrated); during the tracing process, the flies leave their trace based on their movements while "consumeing" each point in the search space; in the middle, the traces of the flies (i.e. new points created from the first ones) are shown, these points will be traced by the flies in the next generation; and on the right, the overall performance of the SDS agents and their attention throughout the sketching process is visualised, thus creating a global map showing where the agents' attention has been focusing on, from the beginning until the end of generating a sketch. In the next generation, the points generated and shown in the middle of Fig. 4 are fed to the system as input and the process is repeated (i.e. SDS's attention mechanism directs DFO flies towards one line at a time to trace, and so forth). 


\section{Autopoiesis}

In 1972, the Chilean biologists, Humberto Maturana and Francisco Varela, coined the term autopoiesis, referring to the self-maintaining chemistry of living cells (Maturana and Varela, 1973). Autopoiesis is composed of two Greek words, 'auto', meaning self, and 'poiesis' meaning production or creation.

There are many ways to think about systems that create products we socially conceive of as art. This research is inspired by Alfred North Whitehead's process view of organisation (Whitehead, 1929), seen though the transformational conceptual-lens of autopoietic theory as stated by Varela and Maturana (1973) according to which we view a creative system as a clearly delineated and identifiable network of continuously operational components producing processes and concomitant elements, bounded as an autonomous entity within its own artistic environment. Maturana and Varela originally define autopoiesis (or self-creation) as follows:

"An autopoietic machine is a machine organized (defined as a unity) as a network of processes of production (transformation and destruction) of components which: (i) through their interactions and transformations continuously regenerate and realize the network of processes (relations) that produced them; and (ii) constitute it (the machine) as a concrete unity in space in which they (the components) exist by specifying the topological domain of its realization as such a network", (p. 78)

On the contrary to autopoiesis, the operation of an allopoietic system is determined in terms of the concatenation of processes. Such processes do not specify the components of the system itself, as a unity; instead the components are produced by other processes that are independent of the organisation of the system. Because the components that make up an allopoietic system's existence are contingent upon other systems, an allopoietic system is never 'fully autonomous'. Some examples of allopoietic systems are: cars, trains and robots.

Furthermore, because an allopoietic system is always contingent on the output of other systems for it existence, its teleology and meaning will always reside in the observers world, never in its own - the systems - world.

To determine whether a system is or is not autopoietic in its organization, Varela, Maturana, and Uribe (1974) have developed six key points or criteria that should be applied to the system; Koskinen (2010) restates these criteria as follows:

(1) Determine, through interactions, if the unity has identifiable boundaries. If the boundaries can be determined, proceed to 2 . If not, the entity is indescribable and we can say nothing.

(2) Determine if there are constitutive elements of the unity, that is, components of the unity. If these components can be described, proceed to 3. If not, the unity is an un-analyzable whole and therefore not an autopoietic system.

(3) Determine if the unity is a mechanistic system, that is, if the component properties are capable of satisfying certain relations that determine the unity, the interactions, and transformations of these components. If this is the case, proceed to 4 . If not, the unity is not an autopoietic system.

(4) Determine if the components that constitute the boundaries of the unity constitute these boundaries through preferential neighbourhood relations and interactions between themselves, as determined by their properties in the space of their interactions. If this is not the case, you do not have an autopoietic unity because you are 
determining its boundaries, not the unity itself. If 4 is the case, however, proceed to 5 .

(5) Determine if the components of the boundaries of the unity are produced by the interactions of the components of the unity, either by transformation of previously produced components, or by transformations and/ or coupling of non-component elements that enter the unity through its boundaries. If not, you do not have an autopoietic unity; if yes, proceed to 6 .

(6) If all the other components of the unity are also produced by the interactions of its components as in 5, and if those which are not produced by the interactions of other components participate as necessary permanent constitutive components in the production of other components, you have an autopoietic unity in the space in which its components exist. If this is not the case and there are components in the unity not produced by components of the unity as in 5 , or if there are components of the unity which do not participate in the production of other components, you do not have an autopoietic unity.

Thus, the successful application of the above six-point taxonomy is sufficient to determine if a system is autopoietically organised (or not).

\section{Autopoietic artist}

The continual creative swarmic processes of our autopoietic artists' attention and reconstitution (sketching) mechanisms are detailed in section 5 of this paper and are illustrated in accompanying video, which displays her behaviour as she artistically decodes a linesketch of an abstract painting made in 1986 (in the style of abstract expressionism) by Willem de Kooning ${ }^{2}$ (Untitled \#2, from Quatre Lithographies).

As observed in section 5, the 'autopoietic' artist is composed of two functionally distinct types of agent: (i) a swarm of attending agents, akin to ants (and governed by the principles of Stochastic Diffusion Search) and (ii) a swarm of drawing agents akin to flies (and governed by the principles of a Dispersive Flies Optimisation). The job of the attending agents is to select areas of meaning ${ }^{3}$ for the drawing agents to 're-interpret'.

Our 'autopoietic' artist is thus continually engaged in a process of sensing her environment and reconstituting it (by iteratively first choosing a line in the scene and re-rendering it). The bounds of the autopoietic artist are defined by the shifting movements of the swarms that comprise her; the elements of the autopoietic artist are the agents of the swarms; the behaviour of each swarm is fully defined by the behaviour of its agents (SDS and DFO); the bounds of the swarms are defined by the hypotheses (positions) of all the SDS agents, whose behaviour changes and in turn modifies the bounds; the components of the boundaries are produced by the interactions of the components of the unity, by transformation of previously produced hypotheses; and because the itera-

\footnotetext{
${ }^{2}$ In our case the artistic environment is initially a sketch of Kooning's abstract canvas, displayed in the middle panel of the video; with the creative output, initially a tabula-rasa, displayed on the left.

${ }^{3}$ For example, in our system we have defined such an area of interest (or 'meaning distinction') to be a line situated in a complex region of the image; a line that has a sharper angle or curve in comparison with other lines. Thus, by suitably redefining the distinction deployed by the population of Stochastic Diffusion agents (as described in Section V), we can modify what constitutes 'meaning' for the autopoietic artist as she interacts with her creative context/environment. The authors in their previous work have used the concept of attention to direct the swarms towards areas of the drawing with higher density, however in this work, identifying sharper angles or curves has been used to define attention. Akin to the complex artist process of creating an artwork, an artist attention is determined by various factors at different times. In the case of the digital artist introduced here, her attention could be impacted by either of the above forms, or both, or a combination of other factors.
} 
tive re-initialisation of the SDS agent-hypotheses are produced by the interactions of the SDS swarm (and all other DFO agents participate as necessary permanent constitutive components in the production of other components), Varela et al's criteria (Varela et al. 1974) for an autopoietic entity are appropriately instantiated in the organisation of our 'autopoietic' artist in the creative space in which her creative unity exists.

Following Luhmann's conception of information processing Luhmann (1995), we view the working autopoietic artist as entailing a reduction in complexity, ravenously consuming 'meaning-distinctions' within her environment; in this way the autopoietic artist iteratively decodes her environment (the De Kooning abstract) by continuously first selecting, then processing, areas of meaning.

Over time, with her artistic 'interest' drawn to areas of rich complexity, the autopoietic artist, so construed, iteratively erases meaningful-distinctions (lines) in her current artistic context, so gradually simplifying the structure of the work. By iteratively focussing on meaning-distinctions as-areas-of-rich-complexity, as the decoding process unfolds it sometimes leads to a less complex (line) structure and ultimately may result in an empty canvas; therein reifying the artwork's 'death' and the tabula rasa (see Fig. 504 This process is attributed to the Gluttonous Autopoietic Artist.

Alternatively, in what is called Contented Autopoietic Artist, by refocussing the autopoietic artist's reflections on 'meaning' (as explored by the Stochastic Diffusion swarm) onto different constitutive elements, and modifying her reconstitution (of the resulting artistic structure), different behaviours of autopoietic creativity can be induced. E.g. by insisting that the re-constitutive processes must generate as many elements of 'meaningdistinction' as they consume, the induced autopoietic processes becomes less likely to fade away and more open-ended in their creative endeavour (see Fig. 65. Figs. 7,8 and 9 show three zoomed sample sketches produced by the autopoietic artist.

In summary, in the context of al-Rifaie and Bishop's 'weak' and 'strong' taxonomy of [computational] creativity (al-Rifaie and Bishop, 2015), although we have presented autopoiesis as offering a new conception of 'strong' artistic creativity ${ }^{6}$, with her 'operational processes' externally instantiated in the execution of a computer program 7 and her idea of 'meaning' (e.g. as areas with lines of sharp corner and curves) externally engineered ${ }^{8}$, sensu-stricto the particular computational autopoieticic artist described herein, fundamentally remains an allopoietic system; we offer her merely as a simple epistemic lens though which to better view, understand and frame the underlying processes of creativity, and not as a computational instantiation of strong creativity. As argued elsewhere (al-Rifaie and Bishop, 2015), any such ontological claim must entail a much more serious engagement with the physical embodiment of the underlying autopoietic system.

\footnotetext{
${ }^{4}$ Link to the video of Gluttonous Swarms: https://youtu.be/aaETyhXh-pQ

${ }^{5}$ Link to the video of Contented Swarms: https://youtu.be/8xc_js7Bos0

${ }^{6}$ The authors have discussed the concept of autopoiesis in other areas such as creativity, art and dance (Bishop and Al-Rifaie, 2016, Bishop and al-Rifaie, 2017).

There is an old debate in the field of computational autopoiesis that effectively rests of the following question: is a computational simulation of an autopoietic entity a genuine autopoietic unity? Those who argue not assert that because the components of, say, the computational autopoietic artist described herein, are fundamentally instantiated via a computer simulation, (whose organisation - power, hardware and software etc - do not participate in regenerating and maintaining).

${ }^{8}$ Contra Maturana and Varela's conception of autopoiesis as a system description to define and explain the nature of living systems as fully autonomous entities, each with a unique teleological behaviour.
} 


\section{Conclusion}

This works deploys two swarm intelligence algorithms: Stochastic Diffusion Search (mimicking the behaviour of ants foraging) and Dispersive Flies Optimisation (mimicking the behaviour of flies hovering over food sources). The former is utilised for facilitating the attention mechanism and the latter is used for regulating the swarmic sketching process. In other words, swarms of ants and flies set off to 'decode' a painting by Willem de Kooning in their own 'swarmic' way. The step-by-step behaviour of the swarms, through the attention and tracing mechanisms is detailed.

Through the concepts behind the attention and tracing mechanisms, the paper focuses on encapsulating the concept of autopoiesis in the behaviour of the autopoietic artist. In other words, the concepts of attention, tracing and autopoiesis are utilised for the rendering purpose where the generated sketches exhibit unique, non-identical output each and every time the swarmic system is set to interpret the line drawings. In terms of the gluttonous autopoietic artist, following each sketching cycle, the complexity of the drawing reduces, until the digital canvas finds itself in its simplest form: the state of emptiness; and in terms of the contented autopoietic artist, the swarms continue to produce non-identical renderings of the original sketch.

Future work will investigate other forms of (visual) attention and tracing mechanisms in the context of autopoiesis as well as generative arts.

\section{References}

al-Rifaie, F. M. and al-Rifaie, M. M. (2016). Maximising Overlap Score in DNA Sequence Assembly Problem by Stochastic Diffusion Search, pages 301-321. Springer International Publishing, Cham.

al-Rifaie, M. M. (2014). Dispersive flies optimisation. In M. P. M. Ganzha, L. Maciaszek, editor, Proceedings of the 2014 Federated Conference on Computer Science and Information Systems, volume 2 of Annals of Computer Science and Information Systems, pages pages 529-538. IEEE.

al-Rifaie, M. M. and Aber, A. (2016). Dispersive flies optimisation and medical imaging. In Recent Advances in Computational Optimization, pages 183-203. Springer.

al-Rifaie, M. M. and Bishop, J. M. (2015). Weak and strong computational creativity'. In Besold and Smaill, editors, Computational Creativity Research: Towards Creative Machines. Springer.

al-Rifaie, M. M. and Bishop, M. (2013a). Swarmic paintings and colour attention. In P. Machado, J. McDermott, and A. Carballal, editors, Evolutionary and Biologically Inspired Music, Sound, Art and Design, volume 7834 of Lecture Notes in Computer Science, pages 97-108. Springer Berlin Heidelberg.

al-Rifaie, M. M. and Bishop, M. (2013b). Swarmic sketches and attention mechanism. In P. Machado, J. McDermott, and A. Carballal, editors, Evolutionary and Biologically Inspired Music, Sound, Art and Design, volume 7834 of Lecture Notes in Computer Science, pages 85-96. Springer Berlin Heidelberg.

al-Rifaie, M. M., Aber, A., and Hemanth, D. J. (2015). Deploying swarm intelligence in medical imaging identifying metastasis, micro-calcifications and brain image segmentation. Systems Biology, IET, 9(6), 234-244.

al-Rifaie, M. M., Cropley, A., Cropley, D., and Bishop, M. (2016). On evil and computational creativity. Connection Science, 28(1).

Aupetit, S., Bordeau, V., Monmarche, N., Slimane, M., and Venturini, G. (2004). Interactive evolution of ant paintings. In The 2003 Congress on Evolutionary Computation, 2003. CEC'03., volume 2, pages 1376-1383.

Bedi, P., Bhasin, V., Mittal, N., and Chatterjee, T. (2014). Fs-sds: Feature selection for jpeg steganalysis using stochastic diffusion search. In 2014 IEEE International Conference on Systems, 
Man, and Cybernetics (SMC), pages 3797-3802. IEEE.

Bishop, J. (1989). Stochastic searching networks. In Proc. 1st IEE Conf. on Artificial Neural Networks, pages 329-331, London, UK.

Bishop, J. M. and Al-Rifaie, M. M. (2016). Autopoiesis in creativity and art. In Proceedings of the 3rd International Symposium on Movement and Computing, MOCO '16, pages 27:1-27:6, New York, NY, USA. ACM.

Bishop, M. and al-Rifaie, M. M. (2017). Autopoiesis, creativity and dance. Connection Science. In press.

Bonabeau, E., Dorigo, M., and Theraulaz, G. (1999). Swarm intelligence: from natural to artificial systems. Oxford University Press, USA.

Bonabeau, E., Dorigo, M., and Theraulaz, G. (2000). Inspiration for optimization from social insect behaviour. Nature, 406, 3942.

Collomosse, J. and Hall, P. (2005). Genetic paint: A search for salient paintings. Applications of Evolutionary Computing, pages 437-447.

Curtis, C. J. (1998). Loose and sketchy animation. In ACM SIGGRAPH 98 Electronic art and animation catalog, page 145.

de Meyer, K., Nasuto, S., and Bishop, J. (2006). Stochastic diffusion optimisation: the application of partial function evaluation and stochastic recruitment in swarm intelligence optimisation. Springer Verlag, 2, Chapter 12 in Abraham, A. and Grosam, C. and Ramos, V. (eds), "Swarm intelligence and data mining".

Greenfield, G. (2005). Evolutionary methods for ant colony paintings. Applications of Evolutionary Computing, Proceedings, 3449, 478-487.

Hertzmann, A. (2001). Paint by relaxation. In Computer Graphics International 2001. Proceedings, pages 47-54. IEEE.

Holldobler, B. and Wilson, E. O. (1990). The Ants. Springer-Verlag.

Kennedy, J. F., Eberhart, R. C., and Shi, Y. (2001). Swarm intelligence. Morgan Kaufmann Publishers, San Francisco ; London.

Koskinen, K. (2010). Autopoietic Knowledge Systems in Project-Based Companies. Springer.

Luhmann, N. (1995). Social systems. Stanford University Press.

Maturana, H. R. and Varela, F. J. (1973). Autopoiesis and cognition: The realization of the living. Number 42. Boston Studies in the Philosophy of Science 42. Dordecht: D. Reidel Publishing Co. ISBN: 90-277-1015-5.

Moglich, M., Maschwitz, U., and Holldobler, B. (1974). Tandem calling: A new kind of signal in ant communication. Science, 186(4168), 1046-1047.

Monmarche, N., Aupetit, S., Bordeau, V., Slimane, M., and Venturini, G. (2003). Interactive evolution of ant paintings. In B. M. e. al, editor, 2003 Congress on Evolutionary Computation, volume 2, pages 1376-1383. IEEE Press.

Moura, L. and Ramos, V. (2007). Swarm paintings-nonhuman art. ARCHITOPIA book, art, architecture and science, pages 5-24.

Salman, A. A., Ahmad, I., and Omran, M. G. (2015). A metaheuristic algorithm to solve satellite broadcast scheduling problem. Information Sciences, 322, 72-91.

Saxe, J. G., Lathen, D., and Chief, B. (1882). The Blind Man and the Elephant. The Poems of John Godfrey Saxe.

Schermerhorn, P. and Scheutz, M. (2009). The impact of communication and memory in hivebased foraging agents. In Artificial Life, 2009. ALife'09. IEEE Symposium on, pages 29-36.

Schlechtweg, S., Germer, T., and Strothotte, T. (2005). Renderbots-multi-agent systems for direct image generation. In Computer Graphics Forum, volume 24, pages 137-148.

Semet, Y., O'Reilly, U. M., and Durand, F. (2004). An interactive artificial ant approach to non-photorealistic rendering. In Genetic and Evolutionary Computation-GECCO 2004, pages 188-200.

Turk, G. and Banks, D. (1996). Image-guided streamline placement. In Proceedings of the 23rd annual conference on Computer graphics and interactive techniques, pages 453-460. ACM.

Urbano, P. (2005). Playing in the pheromone playground: Experiences in swarm painting. Applications on Evolutionary Computing, pages 527-532. 
Urbano, P. (2006). Consensual paintings. Applications of Evolutionary Computing, pages 622632.

Varela, F. G., Maturana, H. R., and Uribe, R. (1974). Autopoiesis: the organization of living systems, its characterization and a model. Biosystems, 5(4), 187-196.

Varela, F. J. and Maturana, H. R. (1973). De máquinas y seres vivos: Una teoría sobre la organización biológica. Santiago de Chile: Editorial Universitaria.

Whitehead, A. N. (1929). Process and reality. an essay in cosmology. gifford lectures delivered in the university of edinburgh during the session 19271928. Macmillan, New York, Cambridge University Press, Cambridge UK.

Zhao, M. and Zhu, S. (2011). Customizing painterly rendering styles using stroke processes. In Proceedings of the ACM SIGGRAPH/Eurographics Symposium on Non-Photorealistic Animation and Rendering, pages 137-146. ACM.

.

.

\footnotetext{
(1)

(1)

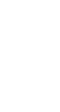



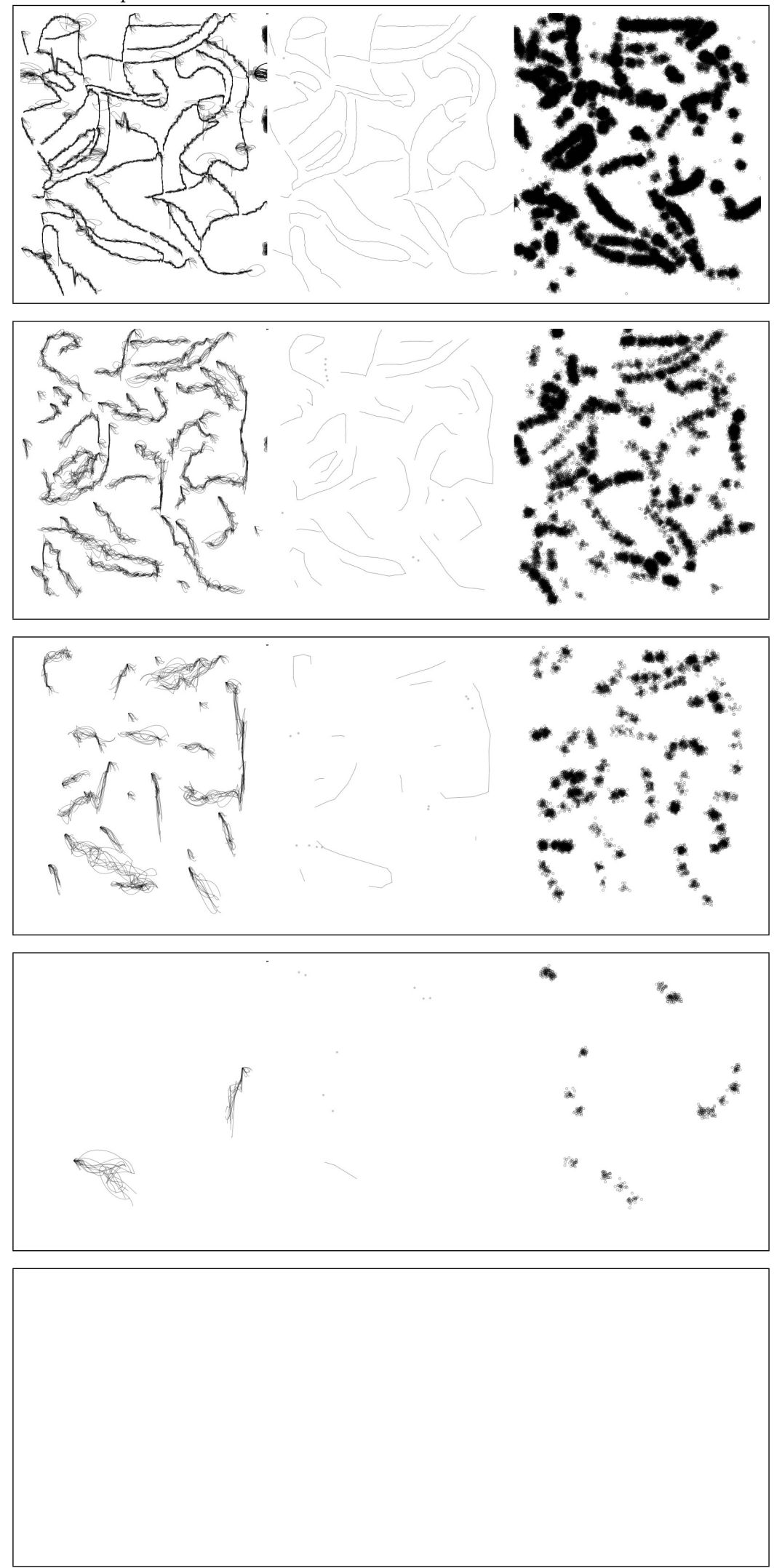

$\begin{array}{lll}\text { (a) Sketch } & \text { (b) Line drawing } & \text { (c) Attention }\end{array}$ 

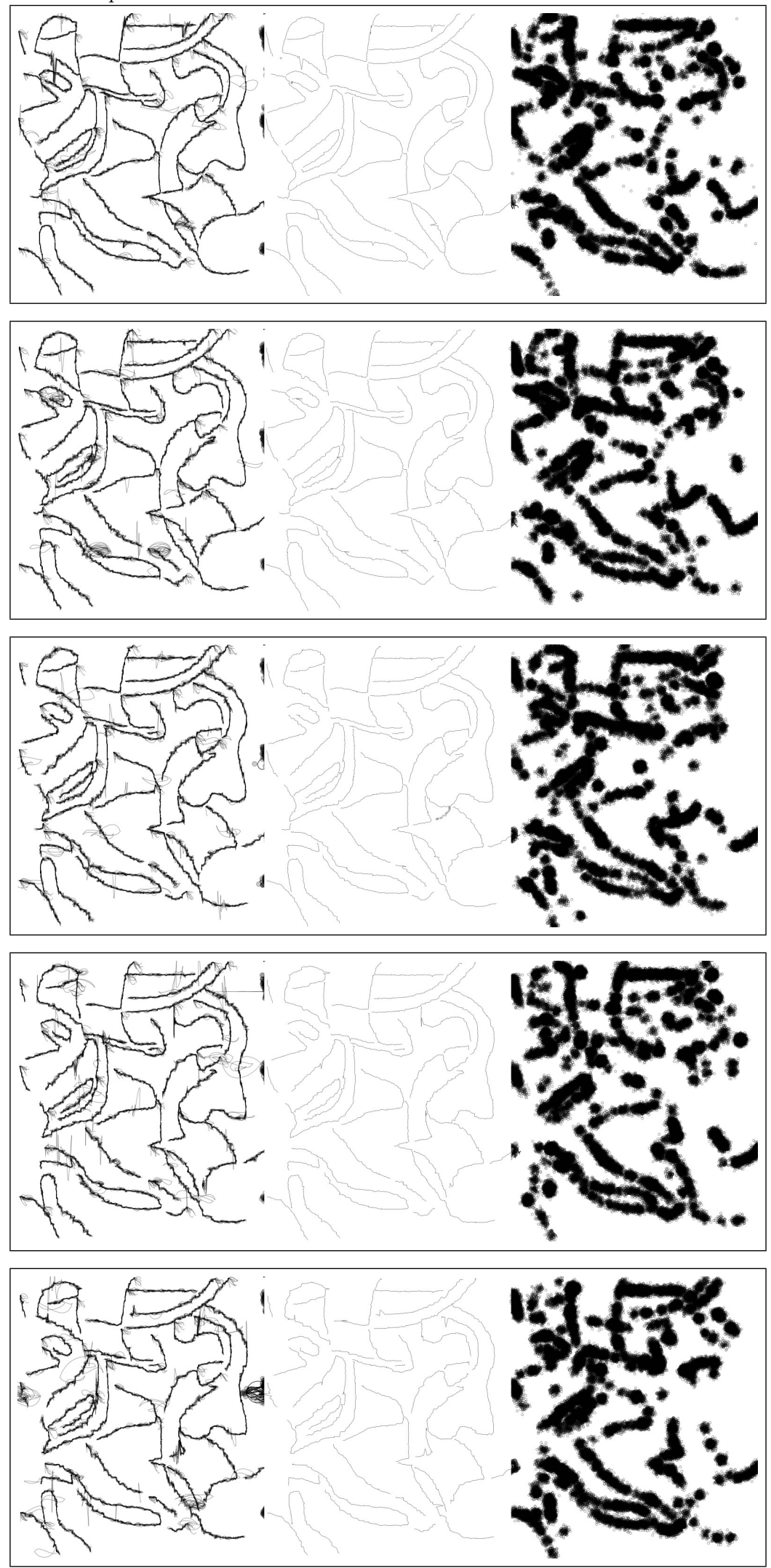

$\begin{array}{lll}\text { (a) Sketch } & \text { (b) Line drawing } & \text { (c) Attention }\end{array}$ 
Figure 7. Sample sketch \#1

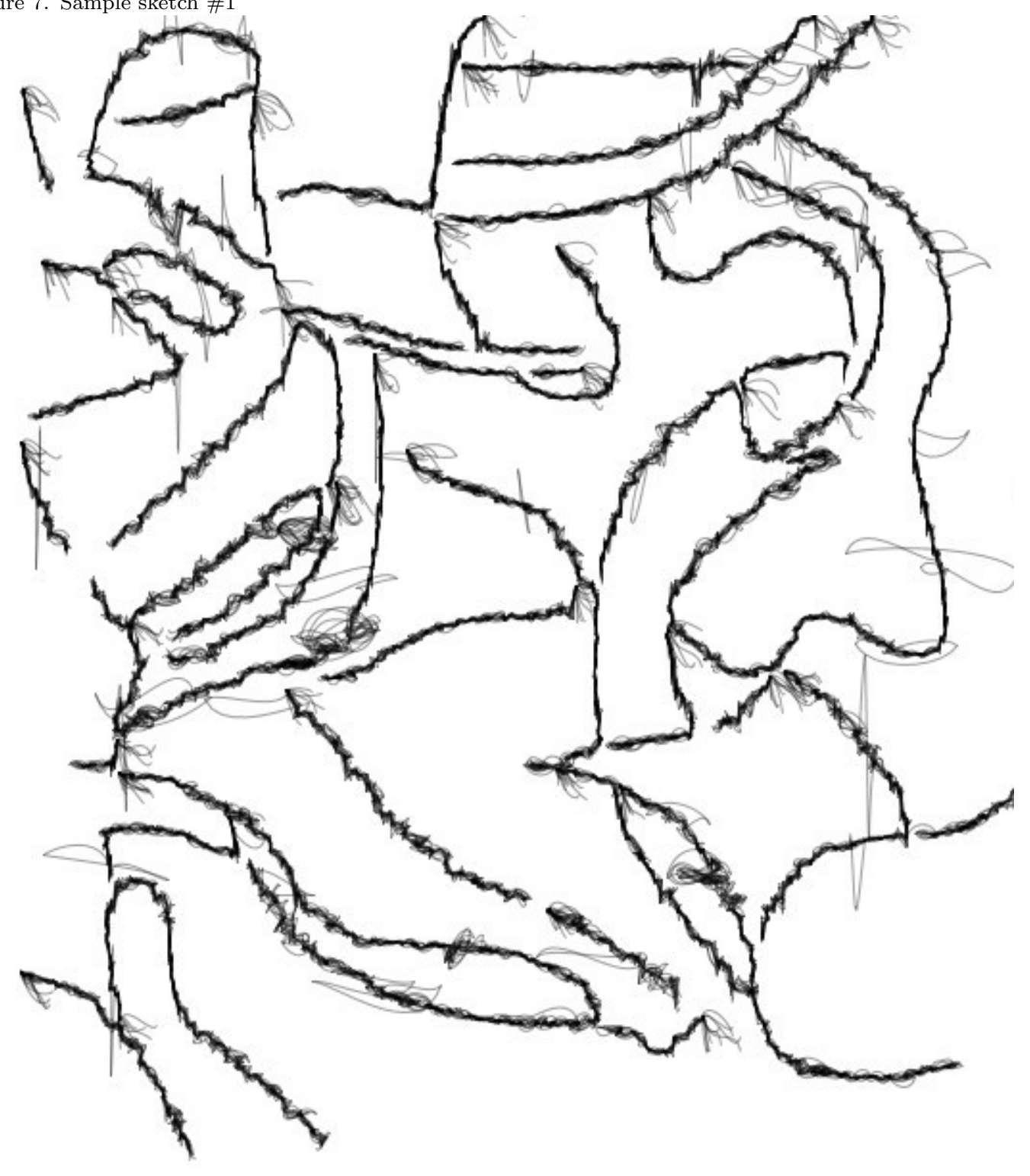


Figure 8. Sample sketch \#2

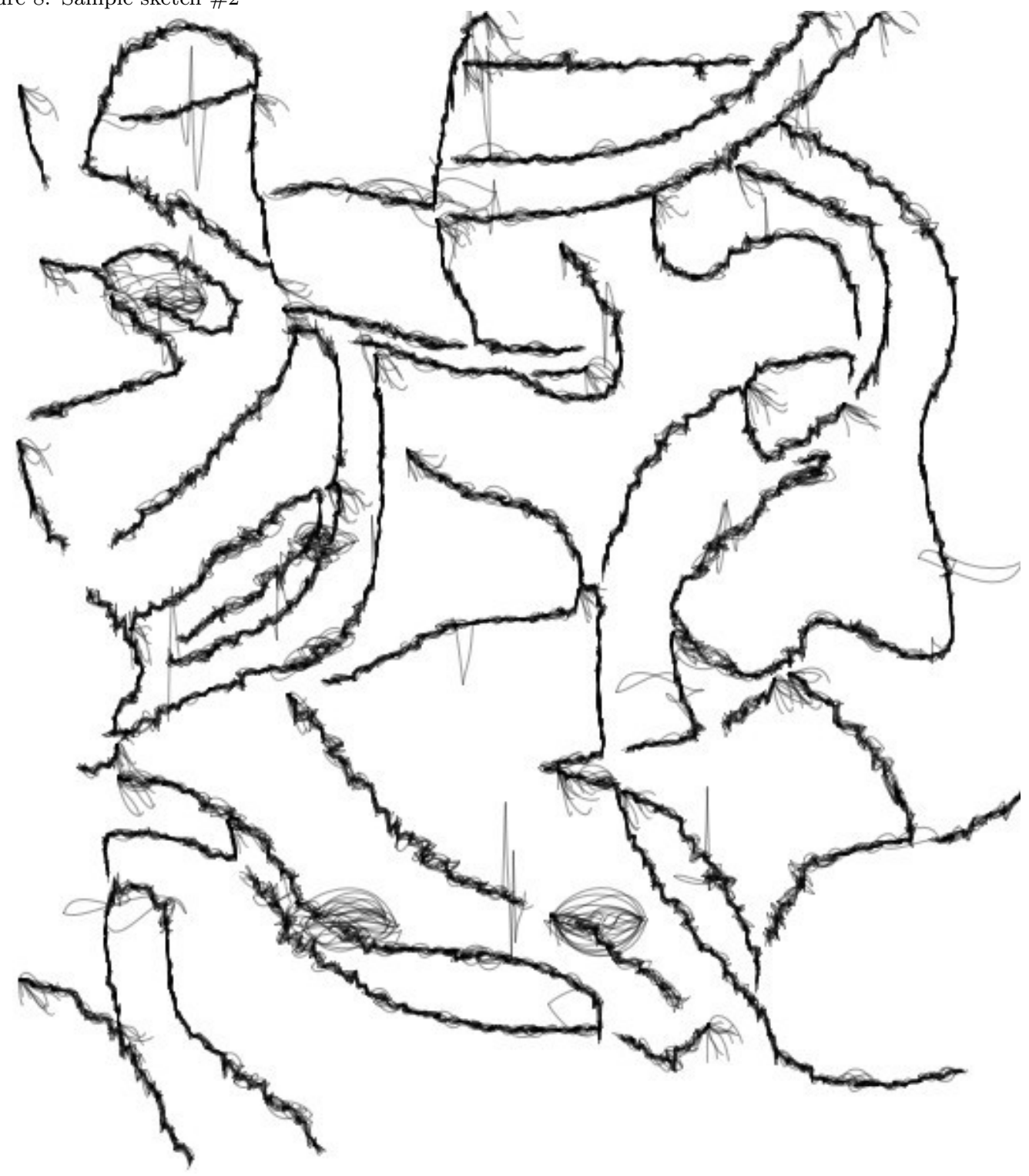


December 15, 2016

Connection Science

ConnScien ${ }^{` 2017 c c}$

Figure 9. Sample sketch \#3

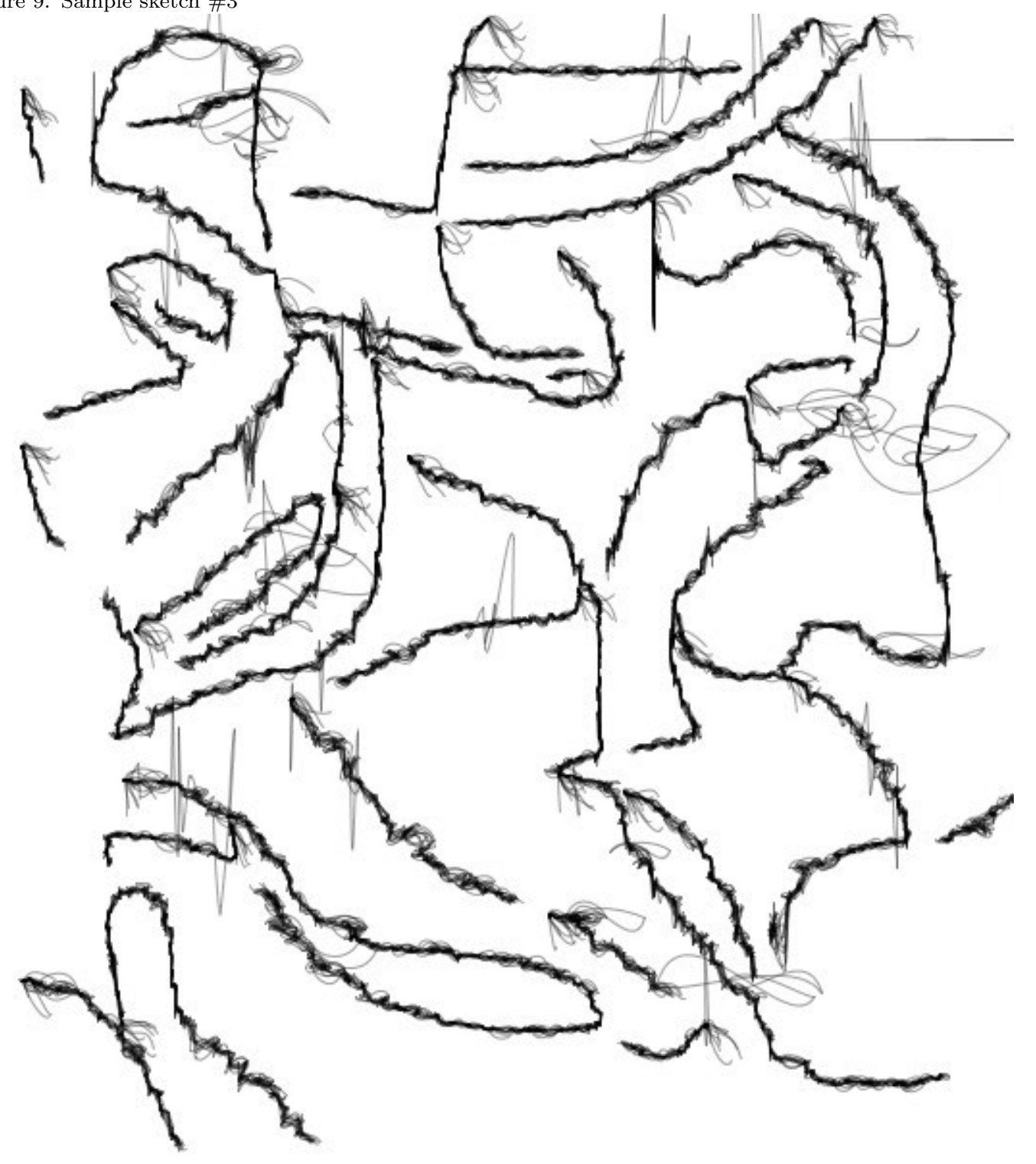

20 